University of Nebraska - Lincoln

DigitalCommons@University of Nebraska - Lincoln

U.S. Department of Veterans Affairs Staff

Publications

U.S. Department of Veterans Affairs

2010

Current Use of Aspirin and Antithrombotic Agents in the United

States Among Outpatients With Atherothrombotic Disease (from

the REduction of Atherothrombosis for Continued Health [REACH]

Registry)

Christopher P. Cannon

Brigham and Women's Hospital and Harvard Medical School, cpcannon@partners.org

Karen E. Rhee

Miriam Hospital

Robert M. Califf

Duke Clinical Research Institute

William Boden

Buffalo General Hospital and State University of New York at Buffalo School of Medicine and Biomedical Sciences

Alan Hirsch

University of Minnesota School of Public Health and Minneapolis Heart Institute Foundation,

hirsc005@umn.edu

Follow this and additional works at: https://digitalcommons.unl.edu/veterans

See next page for additional authors

Cannon, Christopher P.; Rhee, Karen E.; Califf, Robert M.; Boden, William; Hirsch, Alan; Alberts, Mark J.; Cable, Greg; Shao, Mingyuan; Ohman, E. Magnus; Steg, P. Gabriel; Eagle, Kim A.; and Bhatt, Deepak L., "Current Use of Aspirin and Antithrombotic Agents in the United States Among Outpatients With Atherothrombotic Disease (from the REduction of Atherothrombosis for Continued Health [REACH] Registry)" (2010). U.S. Department of Veterans Affairs Staff Publications. 28.

https://digitalcommons.unl.edu/veterans/28

This Article is brought to you for free and open access by the U.S. Department of Veterans Affairs at DigitalCommons@University of Nebraska - Lincoln. It has been accepted for inclusion in U.S. Department of Veterans Affairs Staff Publications by an authorized administrator of DigitalCommons@University of Nebraska - Lincoln. 


\section{Authors}

Christopher P. Cannon, Karen E. Rhee, Robert M. Califf, William Boden, Alan Hirsch, Mark J. Alberts, Greg Cable, Mingyuan Shao, E. Magnus Ohman, P. Gabriel Steg, Kim A. Eagle, and Deepak L. Bhatt 


\title{
Current Use of Aspirin and Antithrombotic Agents in the United States Among Outpatients With Atherothrombotic Disease (from the REduction of Atherothrombosis for Continued Health [REACH] Registry)
}

\author{
Christopher P. Cannon, MD ${ }^{\mathrm{a}, *}$, Karen E. Rhee, MD ${ }^{\mathrm{b}}$, Robert M. Califf, $\mathrm{MD}^{\mathrm{c}}$, \\ William E. Boden, MD ${ }^{\mathrm{d}}$, Alan T. Hirsch, MD ${ }^{\mathrm{e}}$, Mark J. Alberts, MD ${ }^{\mathrm{f}}$, Greg Cable, PhD, MPA ${ }^{\mathrm{g}}$, \\ Mingyuan Shao, MS ${ }^{\mathrm{h}}$, E. Magnus Ohman, MD ${ }^{\mathrm{i}}$, P. Gabriel Steg, MD ${ }^{\mathrm{j}}$, Kim A. Eagle, MD ${ }^{\mathrm{k}}$, and \\ Deepak L. Bhatt, MD, MPH ${ }^{1}$, for the REACH Registry Investigators
}

\begin{abstract}
Despite its proven efficacy, low cost, and wide availability, aspirin remains underused. We examined current aspirin use and determined factors that influence its use among outpatients in the United States (US). The REduction of Atherothrombosis for Continued Health $(\mathrm{REACH})$ Registry is an international, prospective, longitudinal study of $>\mathbf{6 8 , 0 0 0}$ outpatients with established atherothrombosis or $\geq 3$ atherothrombotic risk factors. The rates of aspirin use were compared in various patient subgroups. Multivariate logistic regression models were constructed to determine the factors influencing the baseline use of aspirin and other antithrombotic agents in the US population. Approximately $70 \%$ of 25,686 US outpatients were treated with aspirin, with greater use in the Midwest and among men, whites, and those aged $<65$ years. Among aspirin users, $18 \%$ took other antiplatelet agents and $6 \%$ took oral anticoagulants. Low-dose aspirin ( $\leq 100 \mathrm{mg} /$ day) was used in approximately $2 / 3$ of aspirin users. Of patients not taking aspirin, $1 / 2$ were receiving oral anticoagulants or other antiplatelet agents. However, $15 \%$ of patients used no antithrombotic agent at all. Female gender, current smoking, or having diabetes mellitus were predictors of a lack of antithrombotic use; white race, atrial fibrillation or vascular disease, the use of other risk-reducing medications, or treatment by a cardiologist were associated with a greater likelihood of receiving antithrombotic therapy. In conclusion, approximately $1 / 4$ of US patients with vascular disease are not treated with aspirin for secondary prevention, and $15 \%$ are not treated with any antithrombotic agent. (c) 2010 Elsevier Inc. All rights reserved. (Am J Cardiol 2010;105:445-452)
\end{abstract}

Aspirin irreversibly inhibits the synthesis of thromboxane $\mathrm{A}_{2}$, a potent vasoconstrictor and platelet pro-aggregant. Several trials have shown that aspirin doses of 75 to $150 \mathrm{mg}$

${ }^{a}$ TIMI Study Group, Cardiovascular Division, Brigham and Women's Hospital and Harvard Medical School, Boston, Massachusetts; ' ${ }^{\text {Miriam }}$ Hospital, Providence, Rhode Island; 'Duke Clinical Research Institute, Durham, North Carolina; ${ }^{\mathrm{d} B u f f a l o}$ General Hospital and State University of New York at Buffalo School of Medicine and Biomedical Sciences, Buffalo, New York; ${ }^{\mathrm{e}}$ University of Minnesota School of Public Health and Minneapolis Heart Institute Foundation, Minneapolis, Minnesota; ${ }^{\text {N North- }}$ western University Medical School, Chicago, Illinois; ${ }^{\mathrm{g}}$ Department of Biostatistics, sanofi-aventis, Bridgewater, New Jersey; ${ }^{\mathrm{h} D e p a r t m e n t}$ of Cardiovascular Medicine, Cleveland Clinic, Cleveland, Ohio; ${ }^{\mathrm{i} D u k e}$ University Medical Center, Durham, North Carolina; ${ }^{j}$ INSERM U698 Université Paris 7 AP-HP, Paris, France; ${ }^{k}$ Cardiovascular Center, University of Michigan, Ann Arbor, Michigan; ' Veterans Affairs Boston Healthcare System and Brigham and Women's Hospital, Boston, Massachusetts. Manuscript received June 12, 2009; revised manuscript received and accepted October 7, 2009.

The REduction of Atherothrombosis for Continued Health (REACH) Registry, statistical support, and editorial assistance were supported by the Bristol-Myers Squibb/Sanofi Pharmaceutical Partnership, New York, New York. The REACH Registry has been endorsed by the World Heart Federation, Geneva, Switzerland.

*Corresponding author: Tel: (617) 278-0146; fax: (617) 734-7329.

E-mail address: cpcannon@partners.org (C.P. Cannon). will yield a reduction in cardiovascular events similar to that realized with higher doses of 150 to $325 \mathrm{mg} / \mathrm{day} .{ }^{1}$ In addition, the risk of major bleeding appears to be lower with lower aspirin doses. ${ }^{2}$ Current guidelines recommend 75 to $162 \mathrm{mg} /$ day for secondary prevention of atherosclerotic vascular disease. ${ }^{3}$ No prospective registry data are available describing aspirin use among outpatients with, or at risk of, clinically manifest atherothrombotic disease in a "realworld" setting. The REduction of Atherothrombosis for Continued Health (REACH) Registry, an international, prospective, observational study, was designed to collect data on an ethnically and geographically diverse population across 44 countries in an outpatient setting. The baseline characteristics and 1-year outcomes of the global REACH Registry population have been published previously. ${ }^{4,5}$ The primary objectives of the present analysis were to (1) describe the use of aspirin, including the rates of use and dosage, among patients enrolled in the United States (US) and (2) identify the independent predictors of aspirin use or nonuse in the US.

\section{Methods}

The REACH Registry is an international, prospective, longitudinal study of $>68,000$ patients across 6 continents recruited from December 2003 to June $2004 .{ }^{4-6}$ The present 


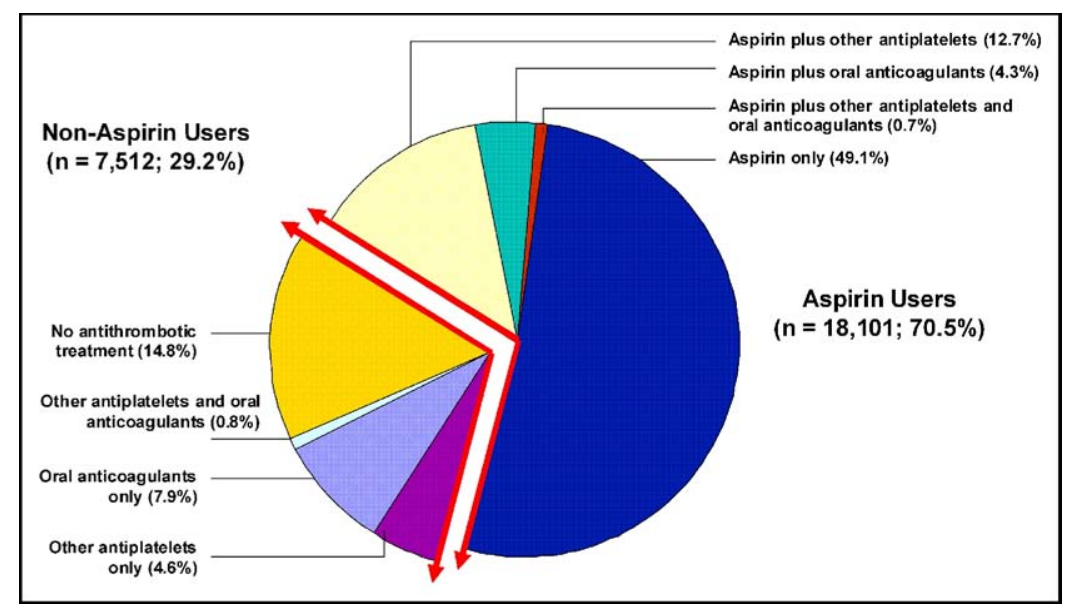

Figure 1. Baseline prevalence of aspirin and other antithrombotic agent use among US patients enrolled in the REACH Registry.

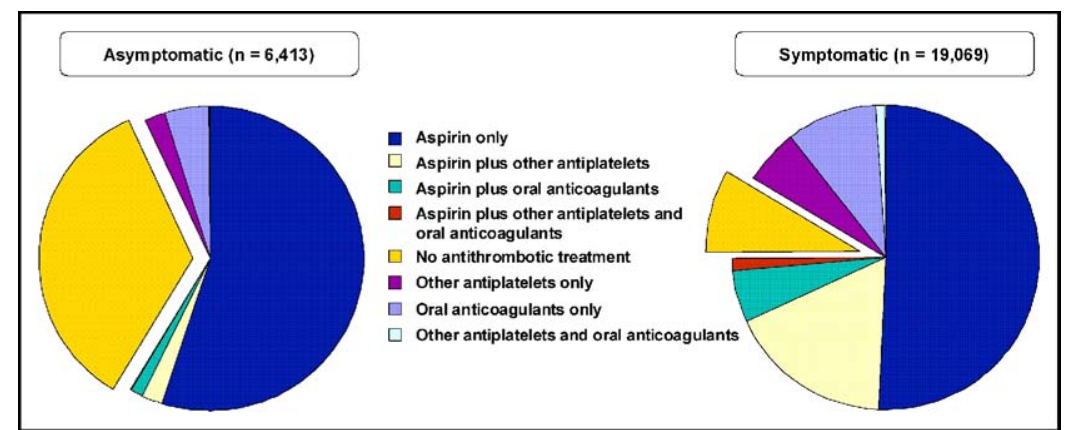

Figure 2. Baseline prevalence of aspirin and other antithrombotic agent use among US patients enrolled in the REACH Registry who were symptomatic or asymptomatic (with $\geq 3$ risk factors only).

report was based on a database lock of July 2006. The methods have been described previously. ${ }^{6}$ In brief, the patients were eligible for enrollment if they were aged $\geq 45$ years and had $\geq 1$ of the following: (1) $\geq 3$ atherothrombotic risk factors; (2) documented coronary artery disease (CAD); (3) documented cerebrovascular disease; or (4) documented peripheral artery disease (PAD). The exclusion criteria included patients who were already participating in other clinical trials, currently hospitalized patients, and those expected to have difficulty returning for follow-up visits. This protocol was submitted to the institutional review board in each country according to the local requirements, and all patients provided signed informed consent. All data were collected locally using a standard subject data form and forwarded to a central site. The data control audits were performed throughout the data collection period by way of site visits conducted at $10 \%$ of all sites that enrolled $\geq 1$ patient (6\% selected randomly, $4 \%$ selected according to performance level). All case report forms at the audited sites were monitored for documentation and accuracy.

The aspirin dosage at baseline was evaluated overall and in patients grouped by age, gender, race, US Census Region, patient type (asymptomatic with risk factors, CAD at baseline, cerebrovascular disease at baseline, PAD at baseline), previous revascularization, previous coronary angioplasty/ stenting, and previous coronary artery bypass grafting without previous revascularization. We also evaluated the dif- ferent combinations of antiplatelet therapy (eg, clopidogrel, ticlopidine) or oral anticoagulant agents used. Data for aspirin users were represented with and without the missing data points for the dosages used. The present report was prepared in compliance with the Strengthening the Reporting of Observational Studies in Epidemiology (STROBE) checklist. $^{7}$

Descriptive values were calculated using the mean, standard deviation (SD), and range for continuous data and counts and/or percentages for categorical data. Bivariate values were also reported as counts and percentages. Multivariate models of the baseline study end points were constructed using the available baseline variables, consisting of patient level data (medical history, risk factors, sociodemographics, and geographic location) and physician data (specialty, years in practice, and geographic location). Contingency tables, simple Poisson and logistic regression models, and graphics were used to assess the magnitude, direction, and functional form of the bivariate relationships between the baseline factors and the study end points of interest. The method of generalized estimating equations was then used to construct multivariate hierarchical Poisson or logistic regression models, as appropriate, given the event proportions and evidence-supporting, technique-specific assumptions. The US Census Region in which the patient resided was used as a nested level in the hierarchical models, and several correlation structures were 
Table 1

Baseline characteristics of United States (US) patients enrolled in the REduction of Atherothrombosis for Continued Health Registry (REACH) according to use of aspirin and other antithrombotic agents

\begin{tabular}{|c|c|c|c|c|c|c|c|c|}
\hline \multirow{2}{*}{$\begin{array}{l}\text { Variable } \\
\text { Concomitant } \\
\text { Antithrombotic } \\
\text { Medication }\end{array}$} & \multicolumn{4}{|c|}{$\begin{array}{l}\text { Aspirin Users } \\
(\mathrm{n}=18,101)\end{array}$} & \multicolumn{4}{|c|}{$\begin{array}{l}\text { Aspirin Nonusers } \\
(\mathrm{n}=7,512)\end{array}$} \\
\hline & $\begin{array}{c}\text { None } \\
(\mathrm{n}=12,612)\end{array}$ & $\begin{array}{c}\text { Other } \\
\text { Antiplatelet } \\
\text { Agents Only } \\
(\mathrm{n}=3,265)\end{array}$ & $\begin{array}{c}\text { Oral } \\
\text { Anticoagulants } \\
\text { Only } \\
(\mathrm{n}=1,103)\end{array}$ & $\begin{array}{c}\text { Both Agents } \\
(\mathrm{n}=189)\end{array}$ & $\begin{array}{c}\text { None } \\
(\mathrm{n}=3,795)\end{array}$ & $\begin{array}{c}\text { Other } \\
\text { Antiplatelet } \\
\text { Agents Only } \\
(\mathrm{n}=11,70)\end{array}$ & $\begin{array}{c}\text { Oral } \\
\text { Anticoagulants } \\
\text { Only } \\
(\mathrm{n}=2,026)\end{array}$ & $\begin{array}{l}\text { Both Agents } \\
(\mathrm{n}=204)\end{array}$ \\
\hline Age (years) & $69.8 \pm 10.2$ & $68.8 \pm 10.6$ & $70.8 \pm 9.9$ & $70.1 \pm 10.5$ & $69.4 \pm 10.7$ & $71.1 \pm 10.3$ & $73.3 \pm 9.6$ & $70.5 \pm 10.5$ \\
\hline \multicolumn{9}{|l|}{ Age group } \\
\hline$<65$ years & $3,913(31.2 \%)$ & $1,179(36.3 \%)$ & $298(27.2 \%)$ & $56(29.9 \%)$ & $1,239(32.8 \%)$ & $325(27.9 \%)$ & $383(18.9 \%)$ & $61(30.0 \%)$ \\
\hline$\geq 65$ years & $8,629(68.8 \%)$ & $2,069(63.7 \%)$ & $799(72.8 \%)$ & $131(70.1 \%)$ & $2,542(67.2 \%)$ & $838(72.1 \%)$ & $1,640(81.1 \%)$ & $142(70.0 \%)$ \\
\hline \multicolumn{9}{|c|}{ 年 } \\
\hline Male & $7,494(59.5 \%)$ & $1,940(59.5 \%)$ & $749(68.0 \%)$ & $118(62.4 \%)$ & $1,743(45.9 \%)$ & $588(50.3 \%)$ & $1,155(57.0 \%)$ & $110(53.9 \%)$ \\
\hline Female & $5,105(40.5 \%)$ & $1,321(40.5 \%)$ & $353(32.0 \%)$ & $71(37.6 \%)$ & $2,051(54.1 \%)$ & $581(49.7 \%)$ & $871(43.0 \%)$ & $94(46.1 \%)$ \\
\hline \multicolumn{9}{|l|}{ Race } \\
\hline White & $10,201(81.7 \%)$ & $2,580(80.0 \%)$ & $942(86.2 \%)$ & $160(84.7 \%)$ & $2,771(73.7 \%)$ & $857(74.1 \%)$ & $1,743(87.0 \%)$ & $171(84.2 \%)$ \\
\hline African-American & $1,215(9.7 \%)$ & $343(10.6 \%)$ & $93(8.5 \%)$ & $14(7.4 \%)$ & $534(14.2 \%)$ & $179(15.5 \%)$ & $157(7.8 \%)$ & $18(8.9 \%)$ \\
\hline Hispanic & $660(5.3 \%)$ & $190(5.9 \%)$ & $37(3.4 \%)$ & $10(5.3 \%)$ & $277(7.4 \%)$ & $68(5.9 \%)$ & $60(3.0 \%)$ & $10(4.9 \%)$ \\
\hline Asian & $339(2.7 \%)$ & $101(3.1 \%)$ & $16(1.5 \%)$ & $3(1.6 \%)$ & $145(3.9 \%)$ & $46(4.0 \%)$ & $28(1.4 \%)$ & $3(1.5 \%)$ \\
\hline Atrial fibrillation & $710(5.7 \%)$ & $240(7.5 \%)$ & $480(44.4 \%)$ & $69(38.1 \%)$ & $181(4.9 \%)$ & $66(5.8 \%)$ & $1,061(53.1 \%)$ & $93(46.3 \%)$ \\
\hline Diabetes & $6,344(50.6 \%)$ & $1,456(44.8 \%)$ & $521(47.5 \%)$ & $92(48.9 \%)$ & $2,377(63.0 \%)$ & $580(49.8 \%)$ & $936(46.6 \%)$ & $97(47.8 \%)$ \\
\hline Current smoking & $1,760(14.3 \%)$ & $457(14.4 \%)$ & $97(8.9 \%)$ & $21(11.4 \%)$ & $666(18.1 \%)$ & $167(14.8 \%)$ & $213(10.9 \%)$ & $25(12.4 \%)$ \\
\hline $\begin{array}{l}\geq 1 \text { Antihypertensive } \\
\text { agent }\end{array}$ & $11,818(93.8 \%)$ & $3,093(94.8 \%)$ & $1,062(96.4 \%)$ & $182(96.3 \%)$ & $3,490(92.2 \%)$ & $1,090(93.2 \%)$ & $1,926(95.1 \%)$ & $191(93.6 \%)$ \\
\hline $\begin{array}{l}\geq 1 \text { Lipid-lowering } \\
\text { agent }\end{array}$ & $10,823(86.0 \%)$ & $2,876(88.4 \%)$ & $916(83.9 \%)$ & $172(91.5 \%)$ & $2,961(78.3 \%)$ & $929(79.6 \%)$ & $1,506(74.5 \%)$ & $173(85.6 \%)$ \\
\hline$\geq 1$ Antidiabetic agent & $5,852(46.5 \%)$ & $1,328(40.8 \%)$ & $483(43.9 \%)$ & $83(44.4 \%)$ & $2,217(58.7 \%)$ & $524(44.9 \%)$ & $825(40.8 \%)$ & $90(44.1 \%)$ \\
\hline Risk factors only & $3,346(26.7 \%)$ & $139(4.3 \%)$ & $78(7.1 \%)$ & $7(3.7 \%)$ & $2,098(56.1 \%)$ & $120(10.4 \%)$ & $279(13.9 \%)$ & $16(7.9 \%)$ \\
\hline Symptomatic & $9,178(73.3 \%)$ & $3,123(95.7 \%)$ & $1,019(92.9 \%)$ & $182(96.3 \%)$ & $1,641(43.9 \%)$ & $1,038(89.6 \%)$ & $1,729(86.1 \%)$ & $187(92.1 \%)$ \\
\hline CVD & $1,890(15.2 \%)$ & $1,097(34.3 \%)$ & $346(32.2 \%)$ & $61(32.6 \%)$ & $439(11.8 \%)$ & $499(43.5 \%)$ & $758(37.9 \%)$ & $92(45.8 \%)$ \\
\hline CAD & $7,798(62.3 \%)$ & $2,463(75.9 \%)$ & $845(77.3 \%)$ & $157(83.5 \%)$ & $1,253(33.6 \%)$ & $665(57.5 \%)$ & $1,244(62.2 \%)$ & $140(69.3 \%)$ \\
\hline PAD & $975(7.7 \%)$ & $466(14.3 \%)$ & $133(12.1 \%)$ & $31(16.4 \%)$ & $236(6.2 \%)$ & $177(15.1 \%)$ & $203(10.0 \%)$ & $42(20.6 \%)$ \\
\hline \multicolumn{9}{|l|}{$\begin{array}{l}\text { Previous } \\
\quad \text { revascularization }\end{array}$} \\
\hline Angio/stent only & $2,307(18.5 \%)$ & $1,099(34.2 \%)$ & $226(20.9 \%)$ & $61(33.3 \%)$ & $287(7.7 \%)$ & $232(20.1 \%)$ & $245(12.3 \%)$ & $45(22.2 \%)$ \\
\hline CABG only & $2,555(20.5 \%)$ & $467(14.5 \%)$ & $259(23.9 \%)$ & $24(13.1 \%)$ & $302(8.1 \%)$ & $149(12.9 \%)$ & $433(21.7 \%)$ & $32(15.8 \%)$ \\
\hline Both & $1,035(8.3 \%)$ & $503(15.7 \%)$ & $183(16.9 \%)$ & $53(29.0 \%)$ & $120(3.2 \%)$ & $107(9.2 \%)$ & $162(8.1 \%)$ & $32(15.8 \%)$ \\
\hline None & $6,576(52.4 \%)$ & $1,144(35.3 \%)$ & $415(37.9 \%)$ & $45(24.3 \%)$ & $3,038(80.7 \%)$ & $669(57.5 \%)$ & $1,151(57.4 \%)$ & $94(46.1 \%)$ \\
\hline \multicolumn{9}{|l|}{ Region } \\
\hline Northeast* & $2,418(19.2 \%)$ & $528(16.2 \%)$ & $201(18.2 \%)$ & $26(13.8 \%)$ & $660(17.4 \%)$ & $224(19.1 \%)$ & $416(20.5 \%)$ & $39(19.1 \%)$ \\
\hline Midwest $^{\dagger}$ & $3,043(24.1 \%)$ & $940(28.8 \%)$ & $296(26.8 \%)$ & $49(25.9 \%)$ & $860(22.7 \%)$ & $266(22.7 \%)$ & $485(23.9 \%)$ & $42(20.6 \%)$ \\
\hline South & $4,791(38.0 \%)$ & $1,245(38.1 \%)$ & $411(37.3 \%)$ & $86(45.5 \%)$ & $1,510(39.8 \%)$ & $469(40.1 \%)$ & $744(36.7 \%)$ & $80(39.2 \%)$ \\
\hline West $^{\S}$ & $2,231(17.7 \%)$ & $529(16.2 \%)$ & $182(16.5 \%)$ & $27(14.3 \%)$ & $721(19.0 \%)$ & $196(16.8 \%)$ & $359(17.7 \%)$ & $41(20.1 \%)$ \\
\hline
\end{tabular}

Data are presented as mean $\pm \mathrm{SD}$ or number $(\%)$.

For each variable, percentages reflect total number of patients with available data only, not total number of patients.

* Maine, New Hampshire, Vermont, Massachusetts, Rhode Island, Connecticut, New York, New Jersey, Pennsylvania.

${ }^{\dagger}$ Ohio, Indiana, Illinois, Michigan, Wisconsin, Minnesota, Iowa, Missouri, North Dakota, South Dakota, Nebraska, Kansas.

${ }^{\ddagger}$ Delaware, Maryland, District of Columbia, Virginia, West Virginia, North Carolina, South Carolina, Georgia, Florida, Kentucky, Tennessee, Alabama, Mississippi, Arkansas, Louisiana, Oklahoma, Texas.

${ }^{\S}$ Montana, Idaho, Wyoming, Colorado, New Mexico, Arizona, Utah, Nevada, Washington, Oregon, California, Alaska, Hawaii.

Angio $=$ angiography CABG $=$ coronary artery bypass grafting; $\mathrm{CVD}=$ cerebrovascular disease.

tested to identify the best fit. The log-likelihood measure, Akaike Information Criterion, Bayesian information criterion, and Hosmer and Lemeshow's statistic were used to assess the various dimensions of model fit. Variance inflation factors, tolerance measures, contingency tables, and graphic methods were used to assess the degree of collinearity and multicollinearity during the model-building process and to facilitate model specification. Only subject data with complete information on model variables were included in the final models. Fisher's exact test was used to compare the categorical variables. No measures were taken to address the potential nonresponse effects of item-level missing data. All statistical hypotheses were tested at $\alpha=0.05$. No adjustments were made for multiple comparisons. Odds ratios (ORs) were calculated with their 95\% confidence intervals. All statistical calculations were performed using SAS, version 8.2 (SAS Institute, Cary, North Carolina). 


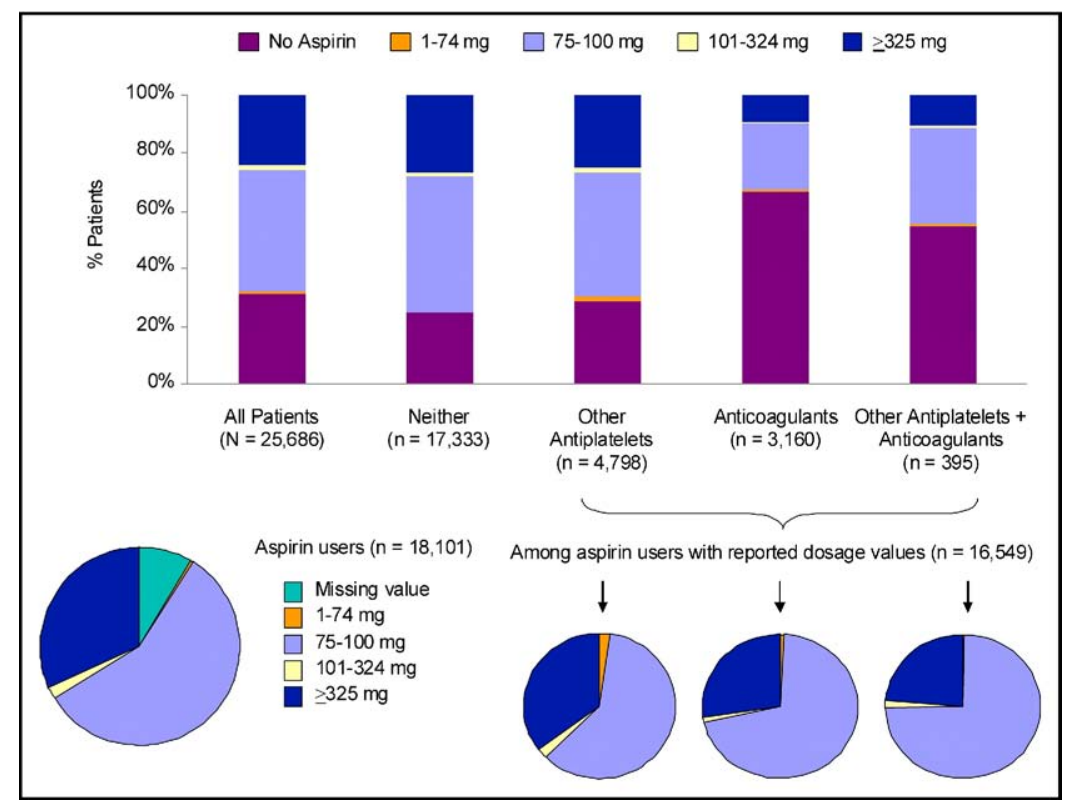

Figure 3. Aspirin use by concomitant antithrombotic medication subgroup in the entire US REACH population and doses used among aspirin users. Bar graph depicts use or lack of aspirin use in the overall population according to concomitant use of other antiplatelet agents and/or oral anticoagulants. Pie charts show dosage breakdown among aspirin users only, accounting for missing data points.

\section{Results}

A total of 25,686 patients were registered in the REACH database from 1,599 practices in the US. At baseline, 15,360 $(59.8 \%)$ had CAD, 5,478 (21.3\%) had cerebrovascular disease, 2,382 (9.3\%) had PAD, and 6,617 (25.8\%) were asymptomatic with risk factors only. Of those with CAD, 11,677 had undergone previous angioplasty/stenting or coronary artery bypass surgery. Of those with cerebrovascular disease, 792 had previously undergone carotid angioplasty/ stenting or carotid surgery. Of the patients with PAD, 1,558 had a history of lower limb angioplasty/stenting/bypass or amputation.

At baseline, $71 \%$ of patients reported taking aspirin (Figure 1). Of these patients, $70 \%$ used aspirin alone, $18 \%$ used aspirin plus other antiplatelet agents, $6 \%$ were taking aspirin and an oral anticoagulant, and $1 \%$ were taking all 3 antithrombotic classes. Among patients not receiving aspirin, $1 / 2$ used no antithrombotic agent, $27 \%$ took oral anticoagulants, $16 \%$ used other antiplatelet agents, and 3\% were taking both another antiplatelet agent and an oral anticoagulant. Predictably, aspirin use was significantly greater among symptomatic patients than among those with risk factors only (75\% vs 59\%; $p<0.001$; Figure 2). Approximately $15 \%$ of patients used no antithrombotic agent at all (Figure 1). In patients with known disease, $25 \%$ were not taking aspirin, among whom $9.1 \%$ were not taking any antithrombotic. In the asymptomatic group, $34.5 \%$ were not taking antithrombotic medication (Figure 2).

The baseline characteristics of the patients, stratified by antithrombotic use, are listed in Table 1. Aspirin use was significantly greater among patients aged $<65$ years than those aged $\geq 65$ years $(73.2 \%$ vs $69.5 \% ; p<0.001)$ and among men versus women (74.4\% vs $65.7 \%$; $\mathrm{p}<0.001)$. Whites had the greatest use of aspirin $(71.6 \%)$, followed by Hispanics (68.6\%), Asians (68.1\%), and African-Americans
$(65.5 \% ; \mathrm{p}=0.017, \mathrm{p}=0.036, \mathrm{p}<0.001$, respectively, compared to whites). The use of aspirin among the US Census Regions was significantly greater in the Midwest $(72.7 \%)$ than in the Northeast $(70.9 \% ; \mathrm{p}=0.039)$, South (70.1\%; $\mathrm{p}<0.001)$, or West $(69.3 \%$; $\mathrm{p}<0.001)$.

Most patients who reported aspirin use at baseline were taking a low dose (75 to $100 \mathrm{mg}$ ) instead of a higher dose $(\geq 325 \mathrm{mg}$ ). Although the total number of those who reported taking aspirin was 18,101 , dosage information was available for only 16,549 patients, among whom nearly $2 / 3$ used aspirin 75 to $100 \mathrm{mg} /$ day (Figure 3 and Table 2). Only $2 \%$ of patients took a "medium" dose (101 to $324 \mathrm{mg}$ ). Patients given the higher dose of aspirin were more often younger, male, and white (Table 2). More patients with CAD took high-dose aspirin compared with those with cerebrovascular disease or PAD (Table 2). Similar to the overall population of aspirin users, patients taking aspirin only (without other antithrombotic agents) primarily used low-dose aspirin (Figure 3). Patients taking aspirin and an oral anticoagulant, with or without other antiplatelet agents, more often used low-dose versus high-dose, aspirin (Figure 3).

With nearly $30 \%$ of patients not taking aspirin, we sought to determine which baseline factors were associated with not using aspirin. The multivariate predictors of not taking aspirin at baseline are plotted in Figure 4. The strongest predictors were the use of oral anticoagulant agents $(\mathrm{OR} 8.32 ; \mathrm{p}<0.0001)$ or other antiplatelet agents (OR 1.62; $\mathrm{p}<0.0001)$; treatment by an endocrinologist (OR 1.38; $\mathrm{p}=$ 0.0002); having diabetes (OR 1.34; $\mathrm{p}<0.0001$ ), taking diabetic medication other than insulin or biguanides (OR $1.32 ; \mathrm{p}=0.0008)$, or taking nonsteroidal anti-inflammatory drugs (OR 1.15; p = 0.0016); female gender (OR 1.37; $\mathrm{p}<0.0001$ ); current smoking (OR 1.19; $\mathrm{p}=0.0004)$; and having congestive heart failure (OR 1.33; $\mathrm{p}<0.0001$ ) or hypertension (OR 1.14; $\mathrm{p}=0.0188$ ). 
Table 2

Aspirin dose of 18,101 United States (US) patients enrolled in the REduction of Atherothrombosis for Continued Health (REACH) Registry reporting aspirin use at baseline

\begin{tabular}{|c|c|c|c|c|c|}
\hline Variable & $\begin{array}{l}\text { Missing or } 0 \mathrm{mg}^{*} \\
\quad(\mathrm{n}=1,552)\end{array}$ & $\begin{array}{l}1-74 \mathrm{mg} \\
(\mathrm{n}=123)\end{array}$ & $\begin{array}{l}75-100 \mathrm{mg} \\
(\mathrm{n}=10,310)\end{array}$ & $\begin{array}{c}101-324 \mathrm{mg} \\
(\mathrm{n}=347)\end{array}$ & $\begin{array}{l}\geq 325 \mathrm{mg} \\
(\mathrm{n}=5,769)\end{array}$ \\
\hline Age (years) & $70.3 \pm 10.5$ & $70.0 \pm 10.2$ & $70.2 \pm 10.3$ & $70.3 \pm 9.8$ & $68.6 \pm 10.2$ \\
\hline \multicolumn{6}{|l|}{ Age group } \\
\hline$<65$ years & $445(29.1 \%)$ & $35(28.9 \%)$ & $3,068(29.9 \%)$ & $111(32.1 \%)$ & $2,117(36.9 \%)$ \\
\hline$\geq 65$ years & $1,086(70.9 \%)$ & $86(71.1 \%)$ & $7,187(70.1 \%)$ & $235(67.9 \%)$ & $3,618(63.1 \%)$ \\
\hline \multicolumn{6}{|l|}{ Gender } \\
\hline Male & $905(58.5 \%)$ & $67(54.9 \%)$ & $5,877(57.1 \%)$ & $239(68.9 \%)$ & $3,760(65.2 \%)$ \\
\hline Female & $642(41.5 \%)$ & $55(45.1 \%)$ & $4,419(42.9 \%)$ & $108(31.1 \%)$ & $2,004(34.8 \%)$ \\
\hline \multicolumn{6}{|l|}{ Race } \\
\hline White & $1,137(74.5 \%)$ & $93(76.9 \%)$ & $8,214(80.5 \%)$ & $311(90.4 \%)$ & $4,816(84.2 \%)$ \\
\hline African-American & $214(14.0 \%)$ & $16(13.2 \%)$ & $1,019(10.0 \%)$ & $20(5.8 \%)$ & $523(9.1 \%)$ \\
\hline Hispanic & $103(6.7 \%)$ & $8(6.6 \%)$ & $589(5.8 \%)$ & $3(0.9 \%)$ & $256(4.5 \%)$ \\
\hline Asian & $57(3.7 \%)$ & $4(3.3 \%)$ & $322(3.2 \%)$ & $7(2.0 \%)$ & $100(1.7 \%)$ \\
\hline Atrial fibrillation & $143(9.4 \%)$ & $11(9.2 \%)$ & $955(9.5 \%)$ & $34(10.1 \%)$ & $425(7.5 \%)$ \\
\hline Diabetes & $784(51.1 \%)$ & $46(37.7 \%)$ & $5,236(51.1 \%)$ & $152(44.7 \%)$ & $2,668(46.4 \%)$ \\
\hline Current smoking & $203(13.6 \%)$ & $22(18.0 \%)$ & $1,348(13.4 \%)$ & $46(13.5 \%)$ & $850(15.0 \%)$ \\
\hline$\geq 1$ Antihypertensive agent & $1,471(95.1 \%)$ & $114(92.7 \%)$ & $9,697(94.2 \%)$ & $321(92.5 \%)$ & $5,435(94.2 \%)$ \\
\hline$\geq 1$ Lipid-lowering agent & $1,326(86.4 \%)$ & $101(82.1 \%)$ & $8,805(85.8 \%)$ & $301(86.7 \%)$ & $5,040(87.6 \%)$ \\
\hline$\geq 1$ Antidiabetic agent & $734(47.7 \%)$ & $44(35.8 \%)$ & $4,842(47.1 \%)$ & $140(40.3 \%)$ & $2,428(42.2 \%)$ \\
\hline Risk factors only & $338(22.0 \%)$ & $16(13.0 \%)$ & $2,608(25.5 \%)$ & $52(15.2 \%)$ & $763(13.3 \%)$ \\
\hline Symptomatic & $1,197(78.0 \%)$ & $107(87.0 \%)$ & $7,632(74.5 \%)$ & $291(84.8 \%)$ & $4,988(86.7 \%)$ \\
\hline Cerebrovascular disease & $380(25.0 \%)$ & $67(55.4 \%)$ & $1,947(19.2 \%)$ & $68(20.0 \%)$ & $1,127(19.9 \%)$ \\
\hline Coronary artery disease & $969(63.3 \%)$ & $53(43.1 \%)$ & $6,316(61.8 \%)$ & $246(71.9 \%)$ & $4,289(74.7 \%)$ \\
\hline Peripheral artery disease & $166(10.7 \%)$ & $12(9.8 \%)$ & $941(9.1 \%)$ & $35(10.1 \%)$ & $536(9.3 \%)$ \\
\hline \multicolumn{6}{|l|}{ Previous revascularization } \\
\hline Angiography/stent only & $270(17.9 \%)$ & $13(10.8 \%)$ & $1,971(19.4 \%)$ & $78(22.7 \%)$ & $1,555(27.3 \%)$ \\
\hline $\begin{array}{l}\text { Coronary artery bypass } \\
\text { grafting only }\end{array}$ & $256(16.9 \%)$ & $16(13.3 \%)$ & $1,856(18.2 \%)$ & $83(24.1 \%)$ & $1,268(22.3 \%)$ \\
\hline Both & $164(10.9 \%)$ & $13(10.8 \%)$ & $929(9.1 \%)$ & $43(12.5 \%)$ & $716(12.6 \%)$ \\
\hline None & $821(53.7 \%)$ & $78(63.9 \%)$ & $5,423(52.9 \%)$ & $140(40.6 \%)$ & $2,148(37.5 \%)$ \\
\hline \multicolumn{6}{|l|}{ Region } \\
\hline Northeast $^{\dagger}$ & $328(21.1 \%)$ & $21(17.1 \%)$ & $1,849(17.9 \%)$ & $66(19.0 \%)$ & $1,112(19.3 \%)$ \\
\hline Midwest & $378(24.4 \%)$ & $42(34.1 \%)$ & $2,500(24.2 \%)$ & $88(25.4 \%)$ & $1,537(26.6 \%)$ \\
\hline South $^{\S}$ & $579(37.3 \%)$ & $40(32.5 \%)$ & $3,926(38.1 \%)$ & $118(34.0 \%)$ & $2,224(38.6 \%)$ \\
\hline West ${ }^{\text {II }}$ & $244(15.7 \%)$ & $19(15.4 \%)$ & $1,931(18.7 \%)$ & $71(20.5 \%)$ & $847(14.7 \%)$ \\
\hline
\end{tabular}

Data are presented as mean \pm SD or numbers $(\%)$.

For each variable, percentage reflects total number of patients with available data only, not total number of patients.

* Of those who checked "yes" to baseline aspirin use, 18 put " 0 " as dose in $\mathrm{mg} /$ day and 1,534 left mg/day empty.

${ }^{\dagger}$ Maine, New Hampshire, Vermont, Massachusetts, Rhode Island, Connecticut, New York, New Jersey, Pennsylvania.

* Ohio, Indiana, Illinois, Michigan, Wisconsin, Minnesota, Iowa, Missouri, North Dakota, South Dakota, Nebraska, Kansas.

${ }^{\S}$ Delaware, Maryland, District of Columbia, Virginia, West Virginia, North Carolina, South Carolina, Georgia, Florida, Kentucky, Tennessee, Alabama, Mississippi, Arkansas, Louisiana, Oklahoma, Texas.

${ }^{\text {II }}$ Montana, Idaho, Wyoming, Colorado, New Mexico, Arizona, Utah, Nevada, Washington, Oregon, California, Alaska, Hawaii.

The factors associated with greater aspirin use at baseline included a history of stent implantation, coronary artery bypass surgery, myocardial infarction, or transient ischemic attack; an ankle-brachial index of $<0.9$; the use of other secondary preventive medications, including angiotensin II receptor blockers, biguanides, angiotensin-converting enzyme inhibitors, nitrates, $\beta$-blockers, statins, and other lipid-lowering agents; and white race (Figure 4).

The multivariate predictors of using low-dose vs higher dose aspirin (Figure 4) included using oral anticoagulants (OR 1.89; $\mathrm{p}<0.0001$ ) or antiplatelets other than aspirin (OR 1.20; $\mathrm{p}<0.0001)$; Asian race (OR 1.76; $\mathrm{p}<0.0001$ ); Hispanic race (OR 1.46; $\mathrm{p}<0.0001$ ); female gender (OR $1.28 ; \mathrm{p}<0.0001$ ); and hypertension (OR 1.14; $\mathrm{p}=0.0202$ ) or diabetes mellitus (OR 1.11; $\mathrm{p}=0.0038$ ). Each additional
10 years of age was associated with a $14 \%$ greater likelihood of taking low-dose aspirin (OR 1.14; $\mathrm{p}<0.0001$ ). For the treating physician, each additional year in medical practice was associated with a $1.2 \%$ greater likelihood of the patient taking low-dose aspirin (OR 1.012; $\mathrm{p}<0.0001)$. Treatment by a physician in a suburban location (compared with an urban or a rural location) was associated with greater use of low-dose aspirin (OR 1.14; $\mathrm{p}=0.0003$ ).

The likelihood of low-dose aspirin use was lower (ie, higher dose aspirin was used) for patients with a history of myocardial infarction (OR 0.89; $\mathrm{p}=0.0037)$, carotid surgery (OR $0.80 ; \mathrm{p}=0.0021)$, ischemic stroke (OR 0.77 ; $\mathrm{p}<0.0001$ ), coronary artery bypass surgery (OR 0.77 ; $\mathrm{p}<0.0001$ ), or stent placement (OR 0.67; $<<0.0001$ ); for 


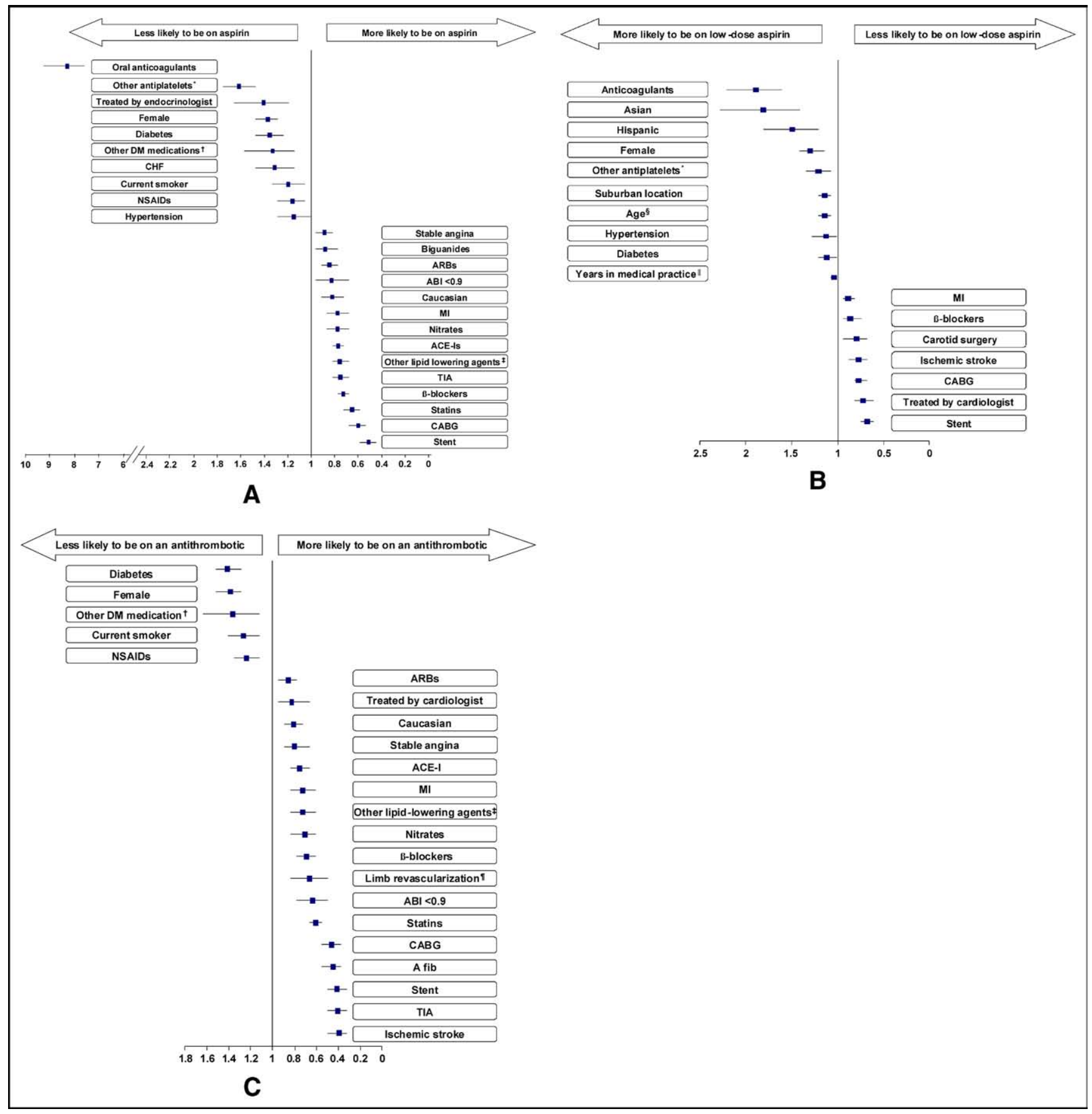

Figure 4. Baseline predictors of $(A)$ lack of aspirin use, $(B)$ low-dose (75 to $100 \mathrm{mg} / \mathrm{day} ;<75 \mathrm{mg}$ was omitted from analysis and $>100 \mathrm{mg}$ was used as referent) aspirin use, and $(C)$ lack of antithrombotic use with $95 \%$ confidence intervals. * Included thienopyridines (clopidogrel and ticlopidine) and dipyridamole, but not nonsteroidal anti-inflammatory drugs or cilostazol. ${ }^{\dagger}$ Included glucosidase inhibitors and meglitinides. ${ }^{\star}$ Included fibrates, bioacid resins, niacin, and ezetimibe. ${ }^{\S}$ For every 10 additional years of age. "History of intermittent claudication associated with lower limb artery angioplasty/stenting/ bypass graft. "For every 1 additional year in clinical practice. ABI = ankle-brachial index; ACE-I = angiotensin-converting enzyme inhibitor; A fib $=$ atrial fibrillation; $\mathrm{ARB}=$ angiotensin II receptor blocker; $\mathrm{CABG}=$ coronary artery bypass graft; $\mathrm{CHF}=$ congestive heart failure; $\mathrm{DM}=$ diabetes mellitus; $\mathrm{MI}=$ myocardial infarction; NSAIDs $=$ nonsteroidal anti-inflammatory drugs; TIA $=$ transient ischemic attack.

those taking $\beta$ blockers (OR 0.85; $\mathrm{p}<0.0001$ ); and for those treated by a cardiologist (OR 0.73 ; p $<0.0001$; Figure 4).

Because many patients not taking aspirin were taking other antithrombotic drugs, we evaluated the multivariate predictors of a lack of any antithrombotic agent (Figure 4).
Patients less likely to be taking any antithrombotic agent had diabetes mellitus (OR 1.41; $<<0.0001$ ), were female (OR 1.38; $\mathrm{p}<0.0001$ ), were current smokers (OR 1.26; $\mathrm{p}$ $<0.0001$ ), and were taking diabetic medications other than insulin or biguanides (OR 1.36; $\mathrm{p}=0.0008$ ) or nonsteroidal anti-inflammatory drugs (OR 1.24; $\mathrm{p}<0.0001$ ). 
Subjects more likely to be taking an antithrombotic agent at baseline were white; had CAD, cerebrovascular disease, atrial fibrillation, $\mathrm{PAD}$, or previous vascular intervention; were taking angiotensin II receptor blockers, angiotensinconverting enzyme inhibitors, nitrates, $\beta$-blockers, statins, or lipid-lowering agents; or were being treated by a cardiologist (Figure 4).

\section{Discussion}

In this large registry of $>25,000$ outpatients in the US with documented vascular disease or multiple risk factors, $70 \%$ of those enrolled were using aspirin. The most common dose of aspirin was 75 to $100 \mathrm{mg} /$ day. This widespread use of low-dose aspirin was supported by the lack of additional clinical benefit ${ }^{1}$ and increased risk of major bleeding ${ }^{2}$ observed with higher doses. Among the aspirin nonusers, nearly $1 / 2$ were taking other antithrombotic agents, although approximately $15 \%$ were not using any antithrombotic therapy. The use for primary prevention was substantially lower than that for secondary prevention. Among patients with symptomatic cardiovascular disease, $25 \%$ were not taking aspirin. Although $63 \%$ of these aspirin nonusers were using a different antithrombotic therapy, $9 \%$ were taking no antithrombotic agent.

The strongest predictor of aspirin nonuse was the use of an oral anticoagulant. This is consistent with a previous study of patients with $\mathrm{CAD}^{8}$ and was not surprising because concomitant use of oral anticoagulants is not necessarily more effective and significantly increases the risk of major bleeding. ${ }^{9-11}$ Despite the lack of improved efficacy and increased risk of bleeding, $6 \%$ of all patients were taking both aspirin and warfarin. Further, $1 \%$ of patients were taking aspirin, warfarin, and another antiplatelet agent, a combination associated with an even greater risk of bleeding. ${ }^{12}$ Perhaps to minimize the risk of bleeding as much as possible, the use of oral anticoagulants was a predictor of low-dose aspirin use. Although it might be expected that the vast majority of the combined aspirin/anticoagulant therapy recipients would have had atrial fibrillation, this was not observed; $<50 \%$ of combined therapy recipients had atrial fibrillation. Overall, these observations suggest a gap between clinical evidence and practice that needs to be addressed.

Current evidence-based clinical practice guidelines, as well as those current at enrollment, have recommended that all patients with a history of CAD, cerebrovascular disease, or PAD, as well as those with a high risk of developing cardiovascular disease, receive aspirin or another antiplatelet therapy as protection against ischemic events. Recent data have suggested that aspirin might not be as effective in preventing ischemic events, suggesting future evidencebased guidelines might not be as liberal in their recommendations for aspirin use, particularly for primary prevention. In the most recent meta-analysis of the efficacy and safety of aspirin for the primary and secondary prevention of vascular disease, the Antithrombotic Trialists' Collaboration concluded that in the current era of secondary prevention, aspirin provides a net benefit for patients with established cardiovascular disease, regardless of gender. ${ }^{13}$ In contrast, they concluded that for primary prevention, aspirin does not provide a net benefit, regardless of gender or underlying disease risk. The investigators of a separate meta-analysis concluded that aspirin used for primary prevention was beneficial in reducing the risk of myocardial infarction in men and stroke in women, with the overall benefit dependent on the baseline risks of cardiovascular disease and gastrointestinal bleeding. ${ }^{14}$ Recent clinical trial data have also shown that aspirin is not effective as primary prevention for patients with asymptomatic PAD with or without diabetes mellitus ${ }^{15,16}$ or as secondary prevention for patients with type 2 diabetes mellitus ${ }^{17}$ or PAD. ${ }^{18}$ It will be of interest to determine whether these new data will shift the use of aspirin in primary prevention.

Our study has several limitations. Efforts were made to recruit representative participants from the patient population of interest; however, the study population was necessarily a judgment/convenience sample, making generalization of the findings beyond the participants difficult. ${ }^{4,5}$ Although the case report form included a large number of relevant variables, important explanatory variables (eg, certain co-morbidities, disease onset and duration, continuity, and quality of care provided) were necessarily omitted to allow the feasibility of this large study and minimize attrition. The patients' history of bleeding events was not collected at baseline. During follow-up, the only bleeding-related variables assessed were the occurrence of hemorrhagic stroke or an episode of bleeding that led to hospitalization and blood transfusion. Specific information about patient adherence was not recorded, suggesting the results might have overestimated the true use of aspirin. Information about the preparation of aspirin (eg, enteric-coated, chewable) and any contraindications to aspirin in aspirin nonusers were not recorded.

Acknowledgment: This report was written and edited by the authors, who take full responsibility for its content. The first draft was written by C. P. Cannon, MD. We wish to thank Susan Abulhawa, MS, and Melanie Leiby, PhD, for their editorial assistance with coordinating the revisions and creating the figures, and Danielle M. Brennan, MS, for her support with statistical analyses. The Cleveland Clinic Coordinating Center for Clinical Research verified all statistical analyses.

1. Antithrombotic Trialists' Collaboration. Collaborative meta-analysis of randomised trials of antiplatelet therapy for prevention of death, myocardial infarction, and stroke in high risk patients. BMJ 2002;324: $71-86$.

2. Serebruany VL, Steinhubl SR, Berger PB, Malinin AI, Baggish JS, Bhatt DL, Topol EJ. Analysis of risk of bleeding complications after different doses of aspirin in 192,036 patients enrolled in 31 randomized controlled trials. Am J Cardiol 2005;95:1218-1222.

3. Smith SC Jr, Allen J, Blair SN, Bonow RO, Brass LM, Fonarow GC, Grundy SM, Hiratzka L, Jones D, Krumholz HM, Mosca L, Pasternak RC, Pearson T, Pfeffer MA, Taubert KA. AHA/ACC guidelines for secondary prevention for patients with coronary and other atherosclerotic vascular disease: 2006 update: endorsed by the National Heart, Lung, and Blood Institute. Circulation 2006;113:2363-2372.

4. Bhatt DL, Steg PG, Ohman EM, Hirsch AT, Ikeda Y, Mas JL, Goto S, Liau CS, Richard AJ, Rother J, Wilson PW. International prevalence, recognition, and treatment of cardiovascular risk factors in outpatients with atherothrombosis. JAMA 2006;295:180-189.

5. Steg PG, Bhatt DL, Wilson PW, D'Agostino R Sr, Ohman EM, Rother J, Liau CS, Hirsch AT, Mas JL, Ikeda Y, Pencina MJ, Goto S. One-year 
cardiovascular event rates in outpatients with atherothrombosis. JAMA 2007;297:1197-1206.

6. Ohman EM, Bhatt DL, Steg PG, Goto S, Hirsch AT, Liau CS, Mas JL, Richard AJ, Rother J, Wilson PW. The REduction of Atherothrombosis for Continued Health (REACH) Registry: an international, prospective, observational investigation in subjects at risk for atherothrombotic events-study design. Am Heart J 2006;151:786.e1-786.e10.

7. Vandenbroucke JP, von Elm E, Altman DG, Gotzsche PC, Mulrow CD, Pocock SJ, Poole C, Schlesselman JJ, Egger M. Strengthening the Reporting of Observational Studies in Epidemiology (STROBE): explanation and elaboration. Ann Intern Med 2007;147:W163-W194.

8. Newby LK, LaPointe NM, Chen AY, Kramer JM, Hammill BG, DeLong ER, Muhlbaier LH, Califf RM. Long-term adherence to evidence-based secondary prevention therapies in coronary artery disease. Circulation 2006;113:203-212.

9. Algra A, De Schryver EL, van Gijn J, Kappelle LJ, Koudstaal PJ. Oral anticoagulants versus antiplatelet therapy for preventing further vascular events after transient ischaemic attack or minor stroke of presumed arterial origin. Cochrane Database Syst Rev 2006;3:CD001342.

10. Anand S, Yusuf S, Xie C, Pogue J, Eikelboom J, Budaj A, Sussex B, Liu L, Guzman R, Cina C, Crowell R, Keltai M, Gosselin G. Oral anticoagulant and antiplatelet therapy and peripheral arterial disease. N Engl J Med 2007;357:217-227.

11. Hurlen M, Abdelnoor M, Smith P, Erikssen J, Arnesen H. Warfarin, aspirin, or both after myocardial infarction. N Engl J Med 2002;347: 969-974.

12. Manzano-Fernández S, Pastor FJ, Marín F, Cambronero F, Caro C, Pascual-Figal DA, Garrido IP, Pinar E, Valdés M, Lip GY. Increased major bleeding complications related to triple antithrombotic therapy usage in patients with atrial fibrillation undergoing percutaneous coronary artery stenting. Chest 2008;134:559-567.
13. Baigent C, Blackwell L, Collins R, Emberson J, Godwin J, Peto R, Buring J, Hennekens C, Kearney P, Meade T, Patrono C, Roncaglioni $\mathrm{MC}$, Zanchetti A. Aspirin in the primary and secondary prevention of vascular disease: collaborative meta-analysis of individual participant data from randomised trials. Lancet 2009;373:1849-1860.

14. Wolff $\mathrm{T}$, Miller T, Ko S. Aspirin for the primary prevention of cardiovascular events: an update of the evidence for the U.S. Preventive Services Task Force. Ann Intern Med 2009;150:405-410.

15. Belch J, MacCuish A, Campbell I, Cobbe S, Taylor R, Prescott R, Lee R, Bancroft J, MacEwan S, Shepherd J, Macfarlane P, Morris A, Jung R, Kelly C, Connacher A, Peden N, Jamieson A, Matthews D, Leese G, McKnight J, O'Brien I, Semple C, Petrie J, Gordon D, Pringle S, MacWalter R. The prevention of progression of arterial disease and diabetes (POPADAD) trial: factorial randomised placebo controlled trial of aspirin and antioxidants in patients with diabetes and asymptomatic peripheral arterial disease. BMJ 2008;337:a1840.

16. Fowkes FGR, Price JF, Stewart MCW, Butcher I, Leng GC, Pell ACH, Sandercock PAG, Fox KAA, Lowe GDO, Murray GD; Aspirin for Asymptomatic Atherosclerosis Trialists. Randomised controlled trial of low dose aspirin in the prevention of cardiovascular events and death in subjects with asymptomatic atherosclerosis. Presented at the European Society of Cardiology, Barcelona, Spain, 2009.

17. Ogawa H, Nakayama M, Morimoto T, Uemura S, Kanauchi M, Doi N, Jinnouchi H, Sugiyama S, Saito Y. Low-dose aspirin for primary prevention of atherosclerotic events in patients with type 2 diabetes: a randomized controlled trial. JAMA 2008;300:2134-2141.

18. Berger JS, Krantz MJ, Kittelson JM, Hiatt WR. Aspirin for the prevention of cardiovascular events in patients with peripheral artery disease: a meta-analysis of randomized trials. JAMA 2009;301:19091919. 